\title{
Menhire in Deutschland
}

\section{Charles-Tanguy Le Roux}

\section{OpenEdition}

\section{Journals}

Édition électronique

URL : http://journals.openedition.org/rao/2785

DOI : $10.4000 /$ rao. 2785

ISBN : 978-2-7535-4053-8

ISSN : 1775-3732

Éditeur

Presses universitaires de Rennes

Édition imprimée

Date de publication : 15 décembre 2014

Pagination : 437-438

ISBN : 978-2-7535-4051-4

ISSN : 0767-709X

\section{Référence électronique}

Charles-Tanguy Le Roux, « Menhire in Deutschland », Revue archéologique de l'Ouest [En ligne], 31 | 2014, mis en ligne le 15 décembre 2014, consulté le 04 décembre 2020. URL : http:// journals.openedition.org/rao/2785; DOI : https://doi.org/10.4000/rao.2785 
Groht Johannes, Schwartz Ralph, Zich Bernd, Meller Harald, 2013 - Menhire in Deutschland, Halle (Saale), Landesamt für Denkmalpflege und Archäologie Sachsen-Anhalt (Landesmuseum für Vorgeschichte), 504 p. (ISBN 978-3-943904-18-5)

437- 438

C'est un bien « beau livre » - en tous les sens du terme - qui vient nous présenter cet aspect du patrimoine mégalithique allemand, beaucoup moins connu en France que son pendant funéraire représenté notamment par les Steinkisten de Hesse ou de Westphalie.

Après une préface de $\mathrm{H}$. Meller, l'ouvrage - impressionnant - s'ouvre par une présentation générale où J. Groht survole différents aspects de la problématique de ces pierres dressées. La comparaison des thèmes abordés avec ceux qui sont de mise en France sur le même sujet est instructive : on y évoque successivement nature et culture, "points d'amour " (en français dans le texte), langage de la nature, hauts lieux sacrés, place dans le paysage, archéoastronomie (à travers Malstein, Dalhausen ou Ahlhorn), christianisation et appropriations tardives (parmi ces dernières, la mise à contribution de sites authentiques ou de forgeries par l'idéologie national-socialiste n'a pas été occultée). Cette introduction débouche tout naturellement sur des "perspectives ", et l'auteur en profite pour exposer sa déontologie de photographe en référence à la pionnière D. Lange. Pour lui, chaque pierre est unique en elle-même comme dans sa relation particulière avec son environnement ; il ne saurait donc être question d'occulter les incongruités qu'engendrent la cohabitation - apparemment guère plus facile Outre-Rhin que chez nous - entre certains mégalithes et les contraintes de notre Xxi siècle; telle est la clé du titre de ce chapitre : In Situ.

Dans les trois chapitres suivants, B. Zich et R. Schwartz abordent fonction, répartition, datation et ornementation du mégalithisme allemand dans un cadre élargi (de l'ouest de la France à la Suisse). C'est ainsi qu'est évoqué tout un art mégalithique, insuffisamment reconnu dans le domaine atlantique malgré sa présentation par Detlef Müller au colloque de Nantes (Müller, 1996) ; les hallebardes de Weilheim y côtoient la hache de combat de Gelnhausen tandis que les visages (finalement assez "parisiens») de Langeneichstädt, Schafstädt ou Rottenburg répondent aux chevrons (très "Petit-Chasseur ») d'Ellenberg ou de Wellen, à côté des « pseudo-gavriniennes » Sonnensteine de Basse-Saxe.

Mais le cœur de l'ouvrage (p. 60 à 491) reste le catalogue des quelque 256 sites retenus. D’emblée, la carte générale (p. 60) montre deux grandes concentrations : au sud-ouest de Mayence, entre Rhin et Moselle d'une part et, bien plus loin vers le nord-est, entre Magdebourg et Erfurt (cette dernière concentration - mal connue chez nous car sur le territoire de l'ancienne RDA - explique l'investissement du Land de Saxe-Anhalt dans cette publication). Un semis irrégulier de pierres dressées couvre par ailleurs tout le nord de l'Allemagne, mais globalement sans relations territoriales claires avec la zone des Steinkisten (malgré le contre-exemple du site de Hekese) ni avec le mégalithisme de l'île de Rügen. Chacun des douze Länder "territoriaux " est cependant concerné, mais de façon très inégale puisque deux d'entre eux, la Rhénanie-Palatinat et la Saxe-Anhalt totalisent, avec respectivement 79 et 55 sites, plus de la moitié de l'effectif par contraste avec de vastes provinces comme la Westphalie (2) ou la Bavière (5). Pour chaque Land, le chapitre s'ouvre par un album de somptueuses photographies issues de la chambre Technika de J. Groht avant les brèves notices consacrées à chacun des sites, selon un plan méthodique qui n'est pas sans évoquer l'Inventaire des Mégalithes de la France. Au fil des pages, on découvre la variété typologique des monuments avec des alignements comme les " 12 apôtres » de Langenbach, des «cercles" plus ou moins importants et même parfois regroupés (le Steintanz de Boitin) ou des complexes plus difficiles à définir (Darmstadt, Derenburg). Les associations à d'autres structures sont illustrées notamment par la trentaine de blocs reliant les deux tombes de Hekese ou les substructions accolées à l'enceinte de Damp.

19 plans (seulement, pourrait-on dire), un peu petits et schématiques mais très lisibles, complètent les notices des sites les plus complexes. En marge de ce catalogue, on relèvera aussi (p. 320) un développement sur les menhirs " aviformes " (7 exemplaires retenus, dont 3 en Rhénanie-Palatinat) et la possible association à un culte chamanique qui en est proposée. Ceci n'est pas sans évoquer la fréquente référence aux oiseaux (an evned en Breton) dans la microtoponymie des menhirs armoricains où certaines pierres font même plus précisément appel au coq, cet oiseau qui fait explicitement référence à l'érection virile dans le parler argotique breton. Reste à savoir s'il y a vraiment communauté d'inspiration entre les deux régions et à quelle(s) époque(s) de telles associations d'idées ont pu se mettre en place...

Tout naturellement, un index bien conçu termine l'ouvrage après une copieuse bibliographie de 289 références, essentiellement en allemand : un rapide survol ne nous en a révélé que trois en anglais, deux en italien et trois en français (si l'on y compte la traduction allemande d'un ouvrage de J.-P. Mohen). Certes, le sujet se devait de faire la part belle aux références monographiques, par nature en langue locale, mais l'appel au " Finistère préhistorique » de Le Pontois 
(1929) semble révélateur d'une certaine faiblesse d'ouverture à l'International... ultimes traces de l'héritage de la RDA ? Dommage également qu'aucune notice biographique - même succincte - ne présente les auteurs.

En résumé, ce somptueux ouvrage est aussi une incontournable mine de renseignements sur tout un pan du mégalithisme européen; il se feuillette avec délectation et se consulte avec grand profit... De quoi inciter à se (re)plonger dans la langue de Goethe tous ceux qui - de près ou de loin - s'intéressent au mégalithisme!

Charles-Tanguy Le Roux 